\title{
Overexpression of miR-146a promotes cell proliferation and migration in a model of diabetic foot ulcers by regulating the AKAP12 axis
}

\author{
Han-Chong Zhang ${ }^{1), 2)}$, Tie Wen ${ }^{1), 2)}$ and Yu-Zhong Cai ${ }^{1), 2)}$ \\ 1) Department of Emergency Medicine, Second Xiangya Hospital, Central South University, Changsha 410011, Hunan Province, P.R. \\ China \\ 2) Emergency Medicine and Difficult Diseases Institute, Second Xiangya Hospital, Central South University, Changsha 410011 , \\ Hunan Province, P.R. China
}

\begin{abstract}
In the current study, we aimed to study the effect of miR-146a on proliferation and migration in an in vitro diabetic foot ulcer (DFU) model by targeting A-kinase-anchoring protein 12 (AKAP12). An in vitro DFU model was initially established using $\mathrm{HaCaT}$ cells derived from human keratinocytes and induced by advanced glycation end products (AGEs). The effects of overexpression of miR-146a on proliferation and migration ability were analysed. The expression levels of miR-146a and AKAP12 were measured by quantitative real-time polymerase chain reaction (qRT-PCR), and AKAP12, hypoxia-inducible factor- $1 \alpha$ (HIF-1 $\alpha$ ), Wnt3a and $\beta$-catenin protein levels were measured by western blotting. The cell proliferation ability was measured by MTT, and the migration ability was analysed by a cell scratch assay. The binding between miR-146a and AKAP12 was identified using a luciferase reporter assay. The results demonstrated that AGEs significantly suppressed cell proliferation and migration, while the expression of miR-146a decreased and the expression of AKAP12 increased. A luciferase reporter assay revealed that miR-146a could directly target AKAP12. Overexpression of miR-146a promoted cell proliferation and migration in an in vitro DFU model and also promoted the expression of HIF-1 $\alpha$, Wnt3a and $\beta$-catenin but suppressed the expression of AKAP12. Co-overexpression of miR-146a and AKAP12 reversed the effect of miR-146a on cell proliferation and migration. Our findings revealed that miR-146a directly targeted AKAP12 and promoted cell proliferation and migration in an in vitro DFU model. This study provides a new perspective for the study of miR-146a in the treatment of DFU.
\end{abstract}

Key words: Mir-146a, A-kinase-anchoring protein 12, $\beta$-catenin, Proliferation, Migration

DIABETIC FOOT ULCERS (DFUs) are caused by diabetic vascular disease, neuropathy, infection and other factors and lead to the destruction of the tissue of the diabetic foot or lower limb [1-4]. In 1999, the World Health Organization (WHO) defined diabetic foot as the infection, ulceration and destruction of deep tissue caused by high blood sugar in the foot. These changes are related to distal nerve abnormalities of the lower extremities and peripheral vascular lesions of different degrees [5-7]. Delayed wound healing has been found in DFU in the

Submitted Mar. 12, 2021; Accepted Jul. 23, 2021 as EJ21-0177

Released online in J-STAGE as advance publication Sep. 4, 2021 Correspondence to: Han-Chong Zhang, Department of Emergency Medicine, Second Xiangya Hospital, Central South University, No. 139 Renmin Road, Furong District, Changsha 410011, Hunan Province, P.R. China \& Emergency Medicine and Difficult Diseases Institute, Second Xiangya Hospital, Central South University, Changsha 410011, Hunan Province, P.R. China.

E-mail: hanchongzhang@esu.edu.cn diabetic mouse model, and impaired wound healing is delayed because of polymorph nuclear neutrophils and macrophages at the wound sites [8]. Neurotensin production by keratinocytes may exert a paracrine effect on other skin cells, namely, fibroblasts, macrophages, and dendritic cells, for correct wound healing [9]. Human keratinocyte $(\mathrm{HaCaT})$ cells were used, maintained at either 10 or $30 \mathrm{mM}$ glucose and treated with or without neurotensin $(10 \mathrm{nM})$. Impaired healing of diabetic wounds is affected by proliferation and migration

Abbreviations: AKAP12, A-kinase-anchoring protein 12; DFU, diabetic foot ulcer; IL-1, interleukin-1; HIF-1 $\alpha$, hypoxia-inducible factor- $1 \alpha$; TNF- $\alpha$, tumour necrosis factor- $\alpha$; DMEM, Dulbecco's modified Eagle medium; AGE, advanced glycation end product; BSA, bovine serum albumin; MTT, 3-(4,5-dimethyl-2-thiazolyl)2,5-diphenyl-2-H-tetrazolium bromide; DMSO, dimethyl sulfoxide; SDS-PAGE, sodium dodecyl sulfate-polyacrylamide gel electrophoresis; PVDF, polyvinylidene fluoride; ECL, enhanced chemiluminescence; qRT-PCR, quantitative real-time polymerase chain reaction 
[10-12]. In wound healing, HaCaT keratinocytes are constantly renewed by proliferation, differentiation and migration in the epidermis to replace worn-out cells due to abrasion [13-15]. In addition, $\mathrm{HaCaT}$ cells are widely used in in vitro models to study the potential mechanism of DFU and wound healing after exposure to high glucose. Therefore, promoting local keratinocyte proliferation and migration of the wound surface could ameliorate the healing disorder of DFU to a certain degree. An in vitro DFU model with $\mathrm{HaCaT}$ cells derived from human keratinocytes was initially established in our study.

MiR-146a has become one of the key regulatory molecules in cell proliferation and migration [16-20] and can be stimulated by different proinflammatory stimuli, such as interleukin-1 (IL-1) [21] and tumour necrosis factor- $\alpha$ $(\mathrm{TNF}-\alpha)$ [22]. Moreover, miR-146a plays an important role in diabetic wound healing [23]. Targeting miR-146a has been used to treat delayed wound healing in human diabetic organ-cultured corneas [23]. However, the downstream mechanism of miR-146a in diabetic foot ulcers is not yet clear. Advanced glycation end products (AGEs) have been proven to be correlated with the development of diabetic complications [24, 25]. Intensive studies have demonstrated that the formation of AGEs may play a role in the pathogenesis of diseases, such as impaired wound healing and diabetic neuropathy, and in diabetic retinopathy [26-28]. AGEs have been used to establish a cell model for the study of DFU and diabetic mesangium $[29,30]$. A-kinase-anchoring protein 12 (AKAP12) has proven to be an essential angiogenic regulator in endothelial cells, playing a vital role in the development of diabetic retinopathy [31]. In our preliminary experiment, through the online analyses of StarBase v2.0 and TargetScan, miR-146a was predicted to have direct binding sites within AKAP12. $\beta$-catenin works as a subunit of the cadherin protein complex, which plays an important role in wound healing via the Wnt signalling pathway $[32,33]$. Studies have demonstrated that the activation of $\beta$-catenin promotes the proliferation and migration of $\mathrm{HaCaT}$ cells in a skin lesion model induced by $\mathrm{H}_{2} \mathrm{O}_{2}$ [34]. AKAP12 regulates human blood-retinal barrier formation by downregulating hypoxia-inducible factor-1 $\alpha$ (HIF-1 $\alpha)$ [35]. In Boso D's study, HIF-1 $\alpha$ was proven to be recruited by the $\beta$-catenin/TCF1 complex to foster neuronal differentiation gene transcription in hypoxic glioblastoma cells [36]. Inhibiting miR-217 can upregulate the HIF-1 $\alpha$ /VEGF pathway to promote angiogenesis and ameliorate inflammation in DFU rats, thereby effectively advancing the healing of ulcerated areas [37]. AKAP12 may affect the Wnt/ $\beta$-catenin pathway by regulating HIF-1 $\alpha$. However, the mechanism of AKAP12 in DFU has not been fully elucidated. To date, there has been no study on the effect of miR-146a on
AKAP12 in an in vitro DFU model.

In this study, we speculated that miR-146a overexpression could suppress AKAP12 and promote cell proliferation and migration in an in vitro DFU model, which was beneficial to wound healing in diabetes. An in vitro DFU model was established with $\mathrm{HaCaT}$ cells derived from human keratinocytes and induced by AGEs. miR-146a was used as the research object of interest to explore the potential effect on cell proliferation and migration by targeting AKAP12 in an in vitro DFU model. This study provides a new perspective for the study of the miR-146a treatment of DFU.

\section{Materials and Methods}

\section{Cell culture and DFU model establishment}

$\mathrm{HaCaT}$ cells (Shanghai Enzyme-linked Biotechnology Co., Ltd., Shanghai, China) were cultured in Dulbecco's modified Eagle medium (DMEM) with high glucose $(4,500 \mathrm{mg} / \mathrm{L})$ or low glucose $(1,000 \mathrm{mg} / \mathrm{L})$, supplemented with $10 \%$ foetal bovine serum (FBS; Invitrogen, Carlsbad, CA, USA) and $1 \%$ antibiotic (100 U/mL penicillin and $100 \mathrm{mg} / \mathrm{mL}$ streptomycin) at $37^{\circ} \mathrm{C}$ and $5 \%$ $\mathrm{CO}_{2}$. The cells grew to an approximately $30 \%$ to $40 \%$ confluence, and then the medium was changed to AGEbovine serum albumin (BSA, Calbiochem, La Jolla, CA) at the same concentration in low glucose DMEM. Cells in logarithmic stage were used in further analysis. To establish a DFU model, HaCaT cell lines in logarithmic stage were divided into a control group, BSA group $(0.1 \mathrm{mmol} / \mathrm{L})$, advanced glycation end products (AGEs) group $(0.1 \mathrm{mmol} / \mathrm{L})$, mannitol group $(40 \mu \mathrm{M})$ and highglucose (H-Glu) group $(40 \mu \mathrm{M})$. BSA was used as a control because AGEs are actually AGE-BSA complexes, and the degree of glycosylation is 70 times greater than that of BSA. HaCaT cells in the AGE group were induced by AGEs. AGEs (AGEs-BSA) were purchased from Beijing Boaosen Biotechnology Co., Ltd. (Beijing, China).

\section{Plasmids construction and cell transfection}

Cell transfections and shRNA interference were performed with Lipofecter liposomal transfection reagent (Beyotime, Shanghai) according to the manufacturer's instructions. The miRNA mimic for miR-146a, miR-146a inhibitor, miRNA negative control, and inhibitor negative control were synthesized by GenePharma (Shanghai, China). Overexpression pcDNA3.1 plasmids were purchased from Thermo Fisher Scientific (Runcorn, Cheshire, UK), and the empty vector was used as a negative control. The AKAP12 sequence was inserted into pcDNA3.1 (Thermo Fisher) to construct an overexpression vector, and the empty vector was used as a negative 
control. The sequences of the primers used for AKAP12 were as follows: forward, 5'-GGAGCCCTAAACAGCC AGGA-3'; reverse, 5'-CTCCTCCTGCCCATCATCTG-3'. For AKAP12 knockdown, AKAP12-targeting shRNAs (GenePharma, Shanghai, China) were synthesized to knockdown the expression of AKAP12 (sh-AKAP12), and a pAdTrack-CMV plasmid was used. A negative control (GenePharma), sh-NC, was used. The infected cells expressing AKAP12, AKAP12 shRNA or sh-NC were divided into AGE, AGE + sh-AKAP12 and AGE + sh-NC groups. The medium was changed $6 \mathrm{~h}$ after transfection with 5 pmol miR-146a mimic, miR-146a inhibitor, mimic negative control, or inhibitor negative control, and after $24 \mathrm{~h}$ of culture, the cells were collected for subsequent experiments. Overexpression and suppression of target genes were confirmed by qRT-PCR after transfection.

\section{Cell proliferation assay by MTT}

The cell proliferation assay was performed with 3(4,5-dimethyl-2-thiazolyl)-2,5-diphenyl-2-H-tetrazolium bromide (MTT, Macklin, Shanghai, China). HaCaT cells were seeded into 96-well plates at a density of $5.0 \times 10^{3}$ cells/well and incubated for $12 \mathrm{~h}$. MTT solution was added to cells at $0,24,48$ and $72 \mathrm{~h}$ after transfection and incubated at $37^{\circ} \mathrm{C}$ in an incubator for $4 \mathrm{~h}$. The supernatant was removed, and then dimethyl sulfoxide (DMSO, Macklin, Shanghai, China) was used to dissolve the formazan in cells, followed by shaking for $10 \mathrm{~min}$. Absorbance values of each sample at $490 \mathrm{~nm}$ were measured with a microplate reader.

\section{Cell scratch assay}

Cell migration was measured by the scratch assay. When the transfected $\mathrm{HaCaT}$ cells were at a confluency of greater than $90 \%$, the tip of a small pipette was used to make a scratch in the plate. The substrate in the lower chamber was DMEM mixed with $10 \%$ FBS. The HaCaT cells migrated across a polycarbonate filter. After $72 \mathrm{~h}$, methanol was used to fix the cells that had moved to the bottom side of the filter for $10 \mathrm{~min}$. Then, the fixed cells were stained with $0.1 \%$ crystal violet. Finally, after 72 h, the scratch width was recorded in an inverted phasecontrast microscope for quantification of the cells.

\section{Quantitative real time polymerase chain reaction (qRT-PCR)}

After $72 \mathrm{~h}$ of transfection, qRT-PCR was conducted to assess the effects of the transfection in each group. Total RNA was extracted from HaCaT cells using an Eastep Super Total RNA Extraction Kit (Promega, Shanghai, China) according to the manufacturer's instructions. Then, the concentration of RNA was determined by a spectrophotometer. For mRNA analysis, a qRT-PCR kit (TaKaRa, Dalian, China) was used for cDNA synthesis, and qRT-PCR was performed to amplify the cDNA template according to the manufacturer's instructions. To analyse the levels of miRNA, the TaqMan MicroRNA RT Kit (Applied Biosystems, San Diego, CA, USA) was used. GAPDH and U6 were used as controls. The data were calculated by $\mathrm{Ct}\left(2^{-\Delta \Delta \mathrm{Ct}}\right)$ method. The sequences of the primers were as follows: miR-146a forward, 5'-CGG CCTGAGAACTGAATTCCA-3' and reverse, 5'-GTCGT ATCCAGTGCAGGGTCCGAGGTAT-3'; AKAP12 forward, 5'-CTGCCTTGGGAGTTTGCC-3' and reverse, 5'GGGTTACGCCTTCCCCAAG-3'; U6 forward, 5'-ATT GGAACGATACAGAGAAGATT-3' and reverse 5'-GGA ACGCTTCACGAATTTG-3'; GAPDH forward, 5'-CCA CAGTCCATGCCATCAC-3' and reverse 5'-GCTTCAC CACCTTCTTGATG-3'.

\section{Luciferase reporter assay}

The wild-type (WT) AKAP12 3'-UTR sequences corresponding to the miR-146a binding sites were inserted into the downstream region of the pGL3-basic vector (SwitchGear Genomics, Shanghai, China) to construct the luciferase reporter WT vector. A mutant (MUT) lacking the seed sequence of the miR-146a binding sites in the AKAP12 3'-UTR was created using a Quik Change Site-Directed Mutagenesis Kit (Agilent, Santa Clara, CA, USA). HaCaT cells were cotransfected with WT or MUT and mimic NC or miR-146a mimic. The transfected $\mathrm{HaCaT}$ cells were collected, and luciferase activity was detected with a dual-luciferase assay kit (Promega).

\section{Western blotting analysis}

Total protein expression in the cells was detected by western blotting. HaCaT cells were collected after transfection and lysed with RIPA lysis buffer (Beyotime, Shanghai, China) on ice. The supernatant was retained to detect its protein concentration after high-speed centrifugation. Protein samples were loaded onto gels for 10\% sodium dodecyl sulfate-polyacrylamide gel electrophoresis (SDS-PAGE) and then transferred to a polyvinylidene fluoride (PVDF) membrane using a semi-dry transfer method. Then, the membrane was incubated with the appropriate primary antibodies $(1: 1,000)$. The membrane was incubated overnight at $4^{\circ} \mathrm{C}$, and then a second antibody was added $(1: 5,000)$ for $1 \mathrm{~h}$. The protein bands were visualized by using an enhanced chemiluminescence (ECL) kit. Blots were repeated at least three times for every condition. After development, the band intensities were quantified using Image-Pro Plus 6.0 analysis software. 


\section{Statistical analysis}

SPSS 19.0 was performed to analyse the data. Data are presented as the mean \pm SD (standard deviation) of at least three independent experiments. The differences between two groups were analysed by Student's $t$-test (two-tailed). The differences among multiple groups $(>2)$ were analysed by one-way analysis of variance (ANOVA).

\section{Results}

\section{MiR-146a expression was decreased and AKAP12 expression was increased in an in vitro $\mathrm{DFU}$ model induced by AGEs and H-Glu treatments}

In an in vitro model of DFU, we compared cell proliferation and migration, as well as the expression of miR-146a and AKAP12, in the induced AGE group and the H-Glu group. The proliferation ability of the AGEtreated and H-Glu-cultured $\mathrm{HaCaT}$ cells was detected by
MTT (Fig. 1A). The results showed that the BSA treatment group showed no significant change in cell proliferation, but the AGE group and $\mathrm{H}-\mathrm{Glu}$ group showed significantly reduced cell proliferation compared with the control group. However, the AGE group and H-Glu group showed no significant differences. Moreover, the scratch wound healing assay also demonstrated that the migration ability of cells in the BSA treatment group was not significantly different from that of the control group, but the AGE group and H-Glu group showed significantly reduced cell migration (Fig. 1B). To investigate the expression of miR-146a and AKAP12 in DFU, we assessed the expression of miR-146a and AKAP12 in HaCaT cells (Fig. 1C-E). The qRT-PCR results showed that the expression of miR-146a was significantly decreased in the AGE group and H-Glu group after $72 \mathrm{~h}$ of treatment (Fig. 1C). Both the qRT-PCR and western blotting results showed that the expression of AKAP12 significantly increased in $\mathrm{HaCaT}$ cells treated with AGEs
A

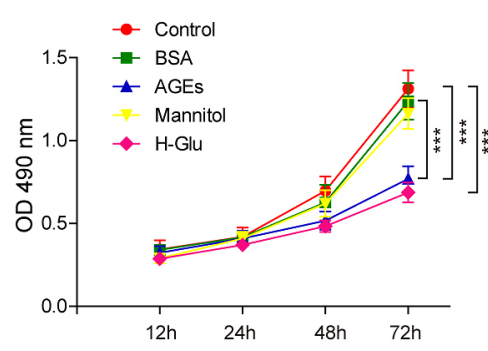

B

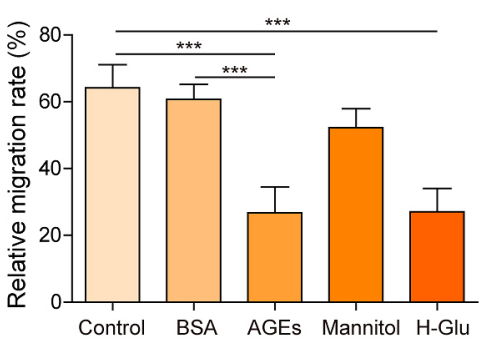

$\mathrm{E}$

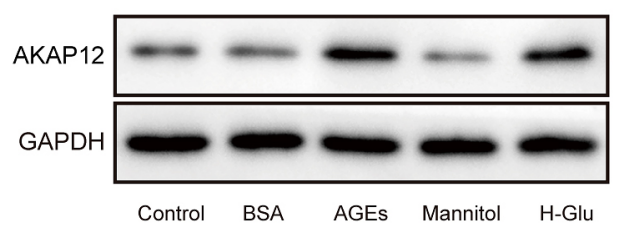

B

C
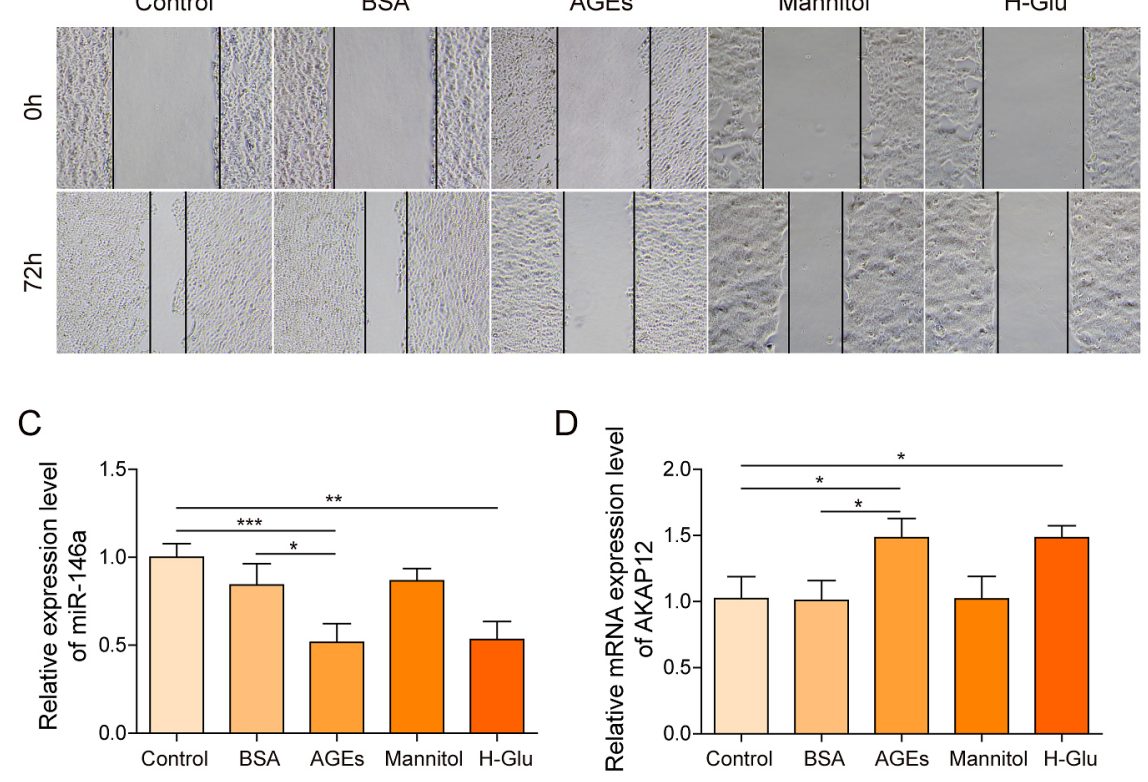

$\mathrm{E}$

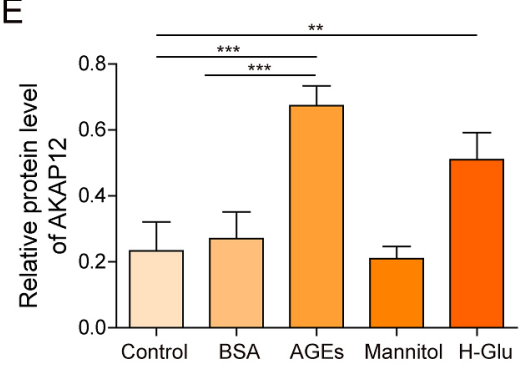

Fig. 1 AGEs and H-Glu reduced the expression of miR-146a and promoted the expression of AKAP12 in an in vitro DFU model. (A) The proliferation of HaCaT cells treated with AGEs and H-Glu for $72 \mathrm{~h}$ was measured by MTT. (B) HaCaT cell migration was measured by a cell scratch assay. (C and D) The expressions of miR-146a and AKAP12 were determined by qRT-PCR in HaCaT cells induced by AGEs and H-Glu treatments. (E) The expression of AKAP12 was measured by western blotting. $n=3 .{ }^{*} p<$ $0.05, * * p<0.01, * * * p<0.001$. 
and H-Glu (Fig. 1D and E). Overall, the expression of miR-146a and the proliferation and migration of $\mathrm{HaCaT}$ cells were reduced, but the expression of AKAP12 was increased in an in vitro DFU model induced by AGE and H-Glu treatments. Both AGE treatment and H-Glu treatment could be used to induce HaCaT cells to construct DFU models in vitro, and there were no significant differences between the two induction methods.

\section{Overexpression of miR-146a promoted cell proliferation and migration in an in vitro $D F U$ model}

To explore whether miR-146a is associated with the process of DFU, we investigated the effect of overexpression of miR-146a on cell proliferation and migration in an in vitro DFU model. As shown in Fig. 2A, we successfully overexpressed miR-146a in an in vitro model of DFU. The proliferation results illustrated that upregulation of miR-146a promoted cell proliferation in an in vitro DFU model (Fig. 2B). The cell migration results demonstrated that the cells overexpressing miR-146a showed a higher migration rate than the control cells (Fig. 2C). Therefore, the high expression of miR-146a promoted cell proliferation and migration in an in vitro model of infected cells in diabetic feet.

\section{MiR-146a directly targeted AKAP12 mRNA}

We overexpressed or suppressed miR-146a expression by transfecting $\mathrm{HaCaT}$ cells with miR-146a mimic or inhibitor, respectively (Fig. 3A). The 3'UTR sequence of $A K A P 12$ was the putative target gene of miR-146a that was predicted by StarBase v2.0 and the TargetScan online database (Fig. 3B). To confirm their association, we generated WT and MUT sequences of the 3'UTR of AKAP12 and transfected them into HaCaT cells (Fig. 3B). MiR-146a directly targeted position 1196-1202 of the AKAP12 3'UTR. The results of the dual-luciferase reporter assay revealed that luciferase activity was significantly decreased by miR-146a overexpression in the WT group and that transfection of the miR-146a inhibitor led to markedly increased luciferase activity, while luciferase activity was not changed in the MUT group (Fig. 3C). In addition, we also analysed the effect of miR-146a on AKAP12, HIF- $1 \alpha$ and the Wnt/ $\beta$-catenin pathway. The miR-146a mimic resulted in a dramatic decrease in the protein levels of AKAP12 and a significant increase in the expressions of HIF-1 $\alpha$, Wnt3a and $\beta$ catenin (Fig. 3D). Consequently, the results showed that the expression of AKAP12 was negatively regulated by miR-146a. AKAP12 may regulate HIF- $1 \alpha$ and also affect the $\mathrm{Wnt} / \beta$-catenin pathway.
A

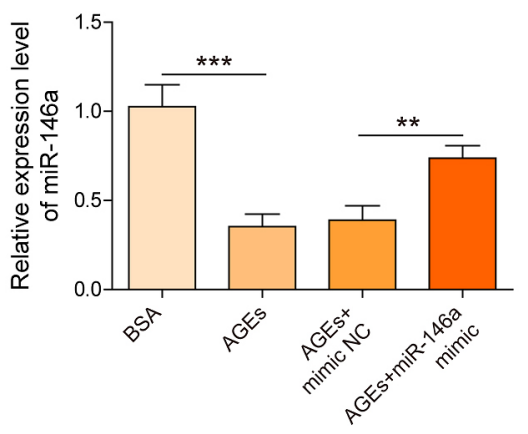

B

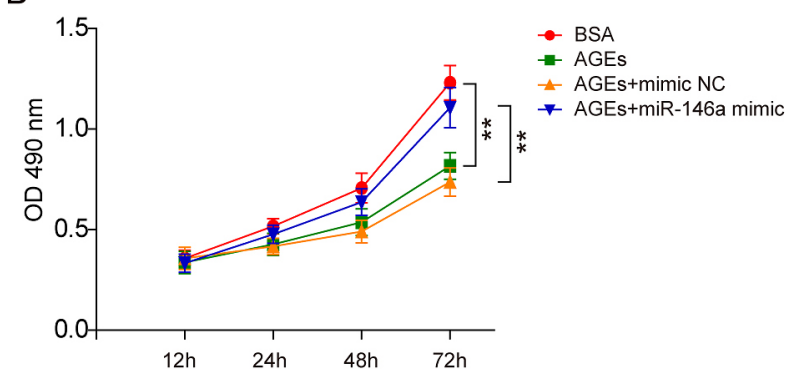

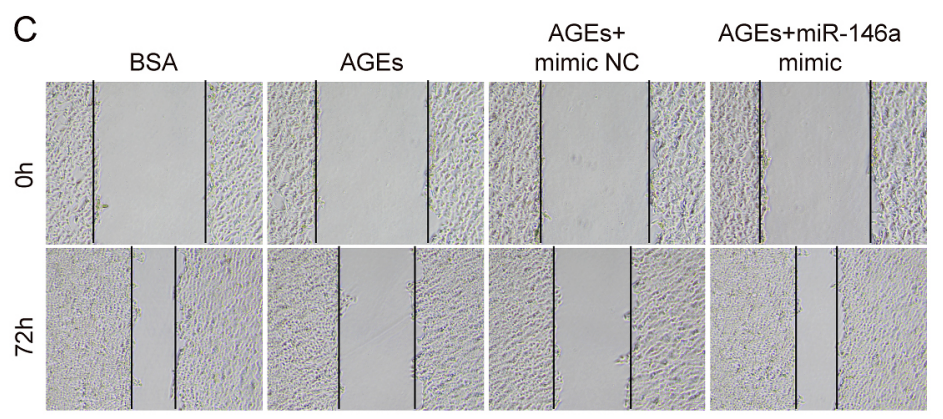

C

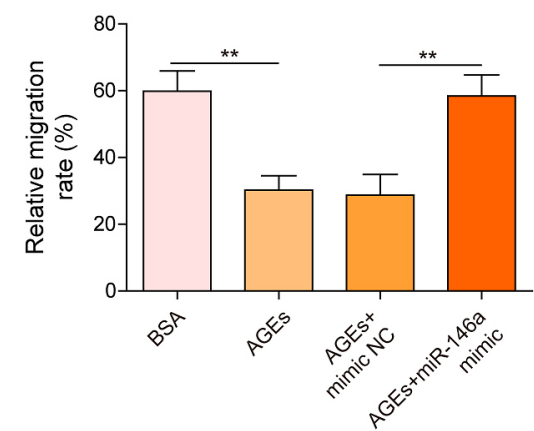

Fig. 2 Overexpression of miR-146a promoted cell proliferation and migration in an in vitro DFU model. (A) The expression of miR-146a was determined by qRT-PCR in HaCaT cells transfected with miR-146a mimic. (B) Cell proliferation was measured using an MTT assay in HaCaT cells transfected with miR-146a mimic. (C) Cell migration was assessed using a cell scratch assay. $n=3$. $* * p<0.01, * * * p<0.001$. 
A

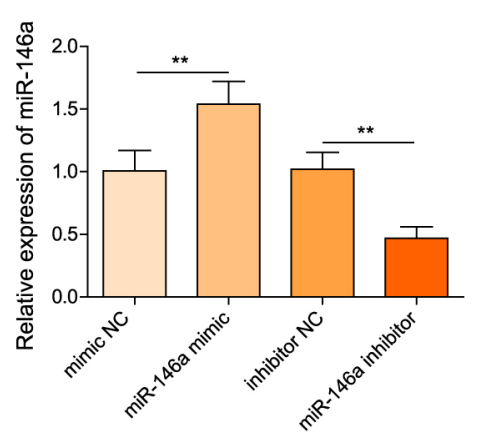

B

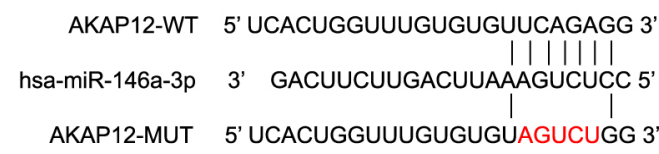

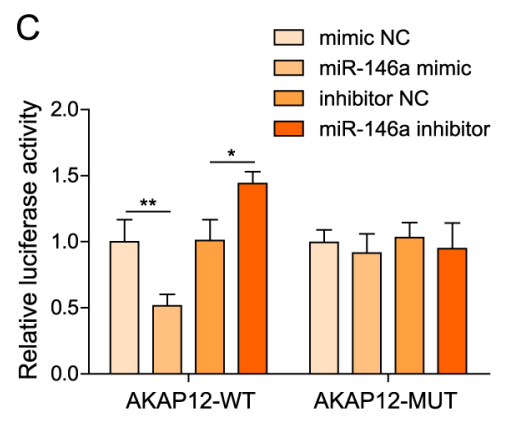

D

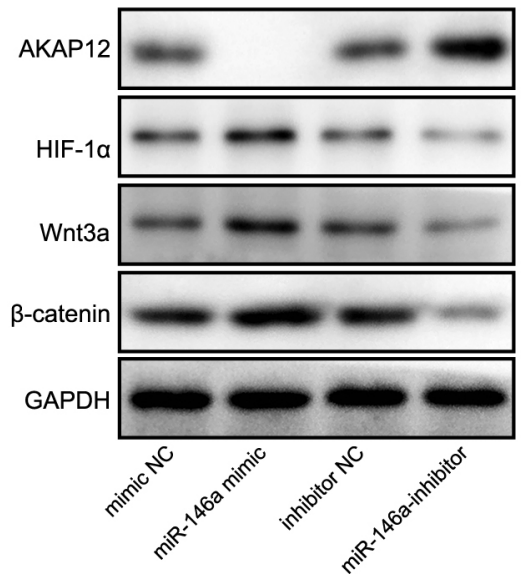

D

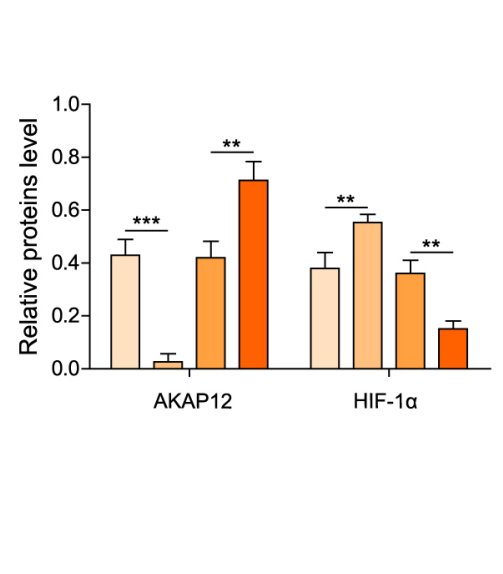

D

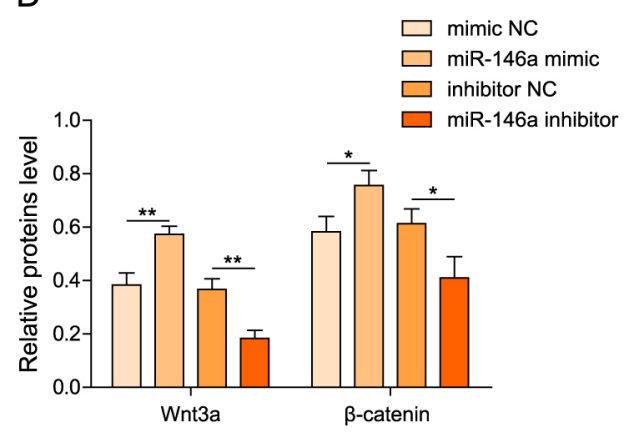

Fig. 3 MiR-146a directly targeted $A K A P 12$. (A) The expression of miR-146a was determined after overexpression or suppression of miR-146a in HaCaT cells by qRT-PCR. (B) The target gene of miR-146a was predicted by the online StarBase v2.0 software. (C) The luciferase activity of miR-146 was assessed by dual-luciferase reporter assay. (D) The expression of AKAP12, HIF-1 $\alpha$, Wnt3a and $\beta$-catenin was assessed by western blotting. $n=3$. $* p<0.05, * * p<0.01, * * * p<0.001$.

\section{Suppression of AKAP12 activated the HIF-1a/Wnt/ $\beta$-catenin axis and promoted cell proliferation and migration in an in vitro DFU model}

To better prove the regulatory relationship of the AKAP12/HIF-1 $\alpha /$ Wnt/ $\beta$-catenin signalling pathway, we knocked down AKAP12 (Fig. 4A). The protein expression levels of HIF-1 $\alpha$, Wnt3a, $\beta$-catenin and AKAP12 were also assessed by western blotting. The protein expression of AKAP12 was significantly suppressed, and the protein expressions of HIF-1 $\alpha$, Wnt3a and $\beta$-catenin were significantly promoted after suppression of AKAP12 (Fig. 4B). The MTT assay results demonstrated that suppression of AKAP12 promoted AGE-induced cell proliferation (Fig. 4C). A cell scratch assay revealed that knocking down AKAP12 promoted the migration ability of $\mathrm{HaCaT}$ cells induced by AGEs (Fig. 4D).

\section{Overexpression of miR-146a suppressed the expression of AKAP12 and promoted cell proliferation and migration in an in vitro $\mathrm{DFU}$ model}

Furthermore, we confirmed the effect of miR-146a on cell proliferation and migration in an in vitro DFU model through AKAP12. HaCaT cells were cotransfected with both a miR-124 mimic and an AKAP12 overexpression vector. The results showed that the expression of miR-146a was significantly increased and that the expression of AKAP12 was decreased when miR-146a was overexpressed; at the same time, AKAP12 expression was significantly increased after transfection with AKAP12, while the expression of miR-146a remained unchanged (Fig. 5A and B). The western blotting results were consistent with those of qRT-PCR (Fig. 5C). When miR-146a was overexpressed, the expression levels of HIF-1 $\alpha$, Wnt3a and $\beta$-catenin were all increased. However, AKAP12 overexpression reversed the expression levels of HIF-1 $\alpha$, Wnt3a and $\beta$-catenin (Fig. 5C). The cell proliferation and migration ability were further analysed. The results suggested that the overexpression of AKAP12 in an in vitro DFU model reversed the effects of overexpression of miR-146a on cell proliferation (Fig. 5D). Similarly, the promoting effects of the miR-146a mimic on cell migration were dramatically reversed by overexpression of AKAP12 (Fig. 5E). 
A

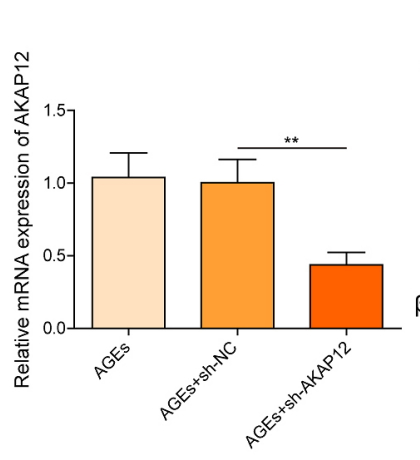

B

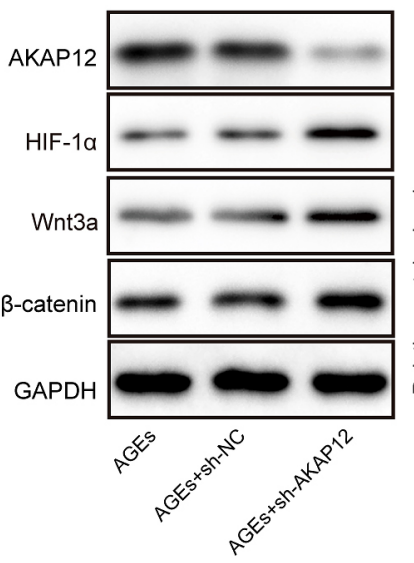

B

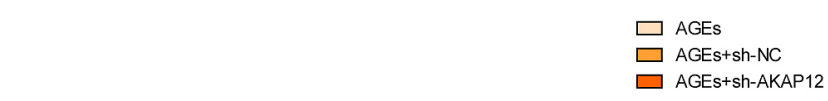

B

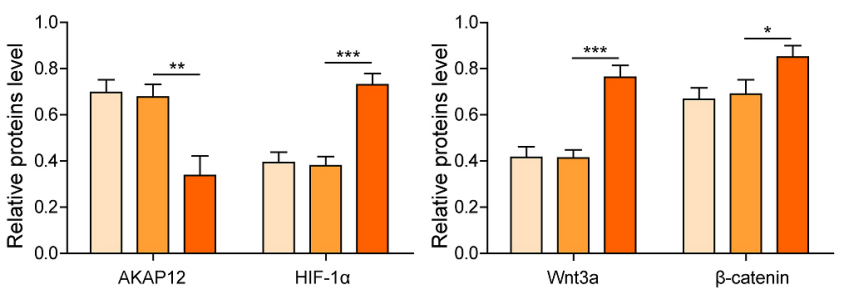

C

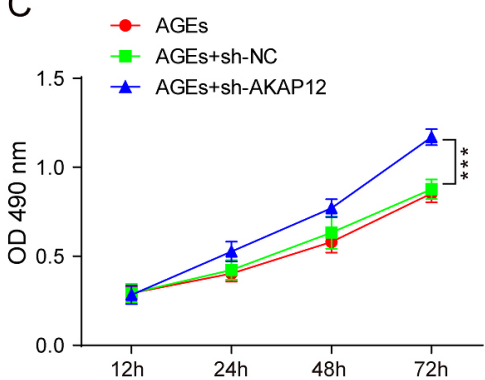

D

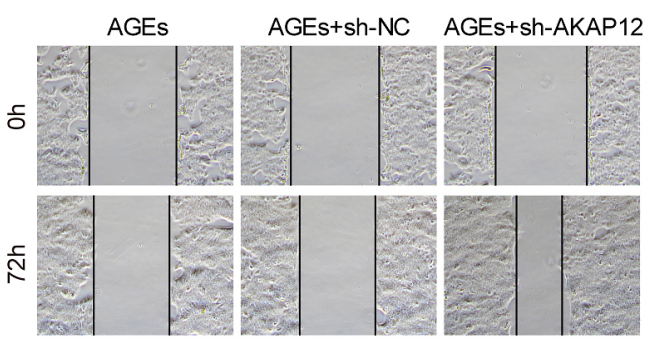

D

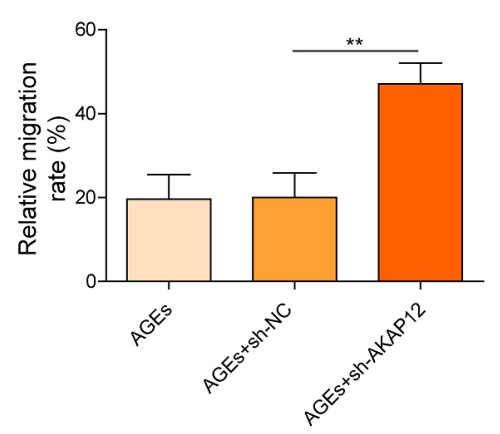

Fig. 4 Suppression of AKAP12 activated the HIF-1 $\alpha /$ Wnt/ $\beta$-catenin axis and promoted cell proliferation and migration in an in vitro DFU model. (A) The mRNA expression of AKAP12 was assessed by qRT-PCR in HaCaT cells after AKAP12 knockdown. (B) The protein expression of HIF-1 $\alpha$, Wnt3a, $\beta$-catenin and AKAP12 was assessed by western blotting. (C) The cell proliferation of HaCaT cells induced by AGEs was analysed by MTT. (D) The migration ability of HaCaT cells induced by AGEs by cell scratch assay. $n=3 . * p<0.05, * * p<0.01, * * * p<0.001$.

Therefore, overexpression of miR-146a suppressed the expression of AKAP12 and increased HIF-1 $\alpha$, Wnt3a and $\beta$-catenin expressions, promoted cell proliferation and migration ability and increased diabetic infected wound healing ability in an in vitro DFU model. We speculate that AKAP12 might affect the Wnt/ $\beta$-catenin pathway by regulating HIF-1 $\alpha$ in the development of DFU.

\section{Discussion}

Patients with DFU commonly experience ischaemia, diabetic vascular disease, neuropathy, and infection, which often leads to amputation of the leg and has a high rate of mortality and recurrence [1-3]. In our study, our findings first demonstrated that miR-146a could directly target AKAP12. Overexpression of miR-146a promoted cell proliferation and migration in an in vitro model of DFU by suppressing AKAP12, and its regulatory mechanism might be related to the activation of the HIF- $1 \alpha /$ Wnt/ $\beta$-catenin signalling pathway.
As one of the key regulatory molecules in cell proliferation and migration, miR-146a is involved in various diseases, such as diabetic wound-healing impairment, breast cancer, liver cancer, acute leukaemia and diabetes $[16,17,20,38,39]$. It can be stimulated by different proinflammatory stimuli, such as interleukin-1 (IL-1) and tumour necrosis factor- $\alpha(\mathrm{TNF}-\alpha)[21,22]$. Moreover, it is downregulated in diabetic mouse wounds [16]. Both miR-145 and miR-155 induce similar phenotypes to miR-146a. However, analyses by StarBase v2.0 and the TargetScan online database predicts no direct binding sites for miR-155 in AKAP12, and the possible mechanism of miR-155 in diabetic wound healing has been reported [16]. The role of miR-145 in wound healing is not very certain, except for the differential expression of miR-145 [16]. However, the possible regulatory mechanism of miR-145 in the diabetic foot is to be explored next in our further studies. In contrast, we aimed to explore the potential mechanism of miR-146a in DFU in our study. The results revealed that miR-146a expression 
A

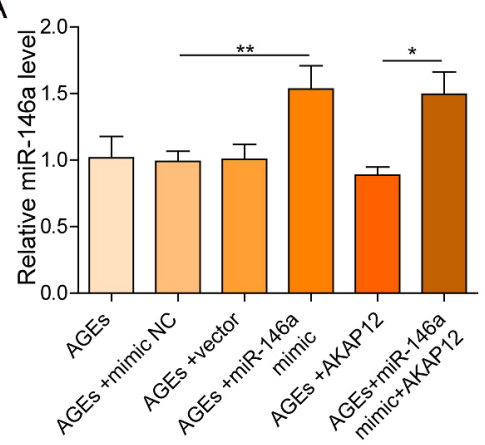

C

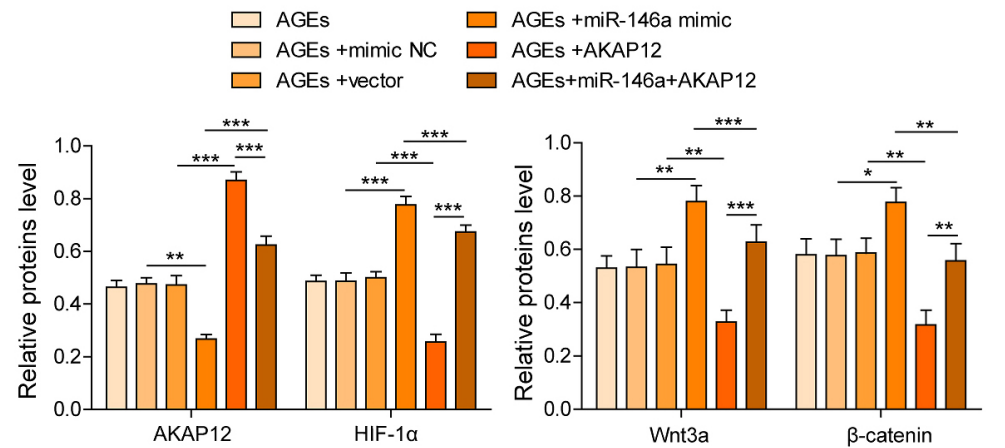

C
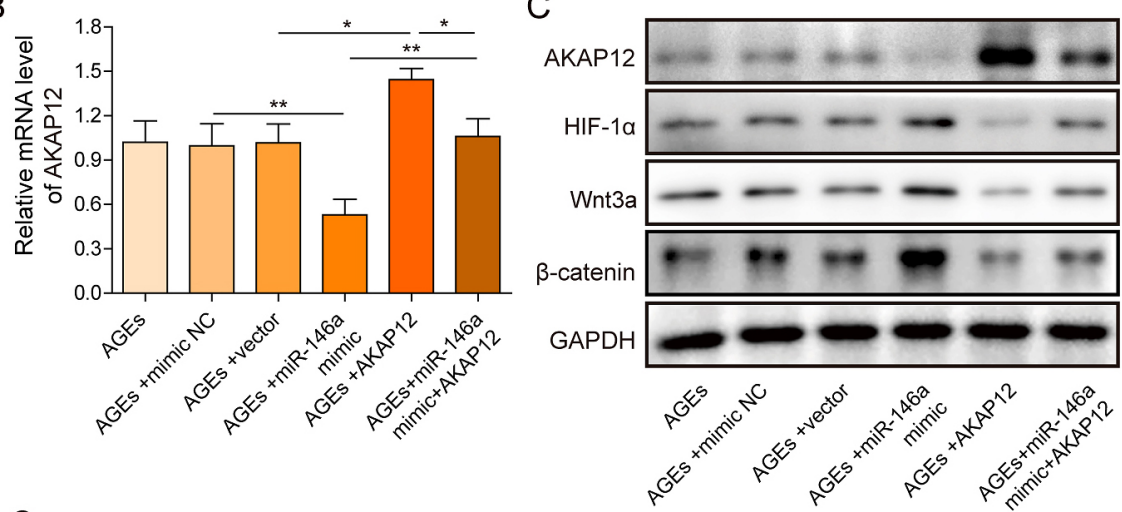

D

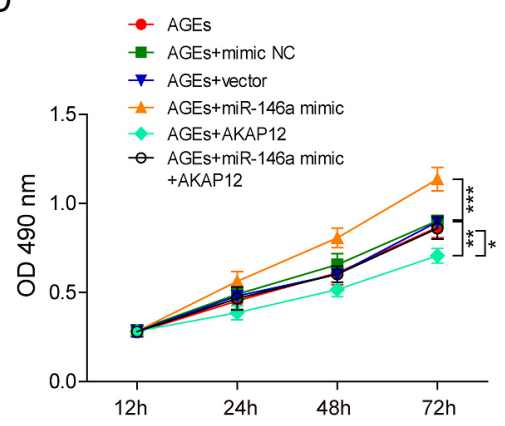

E

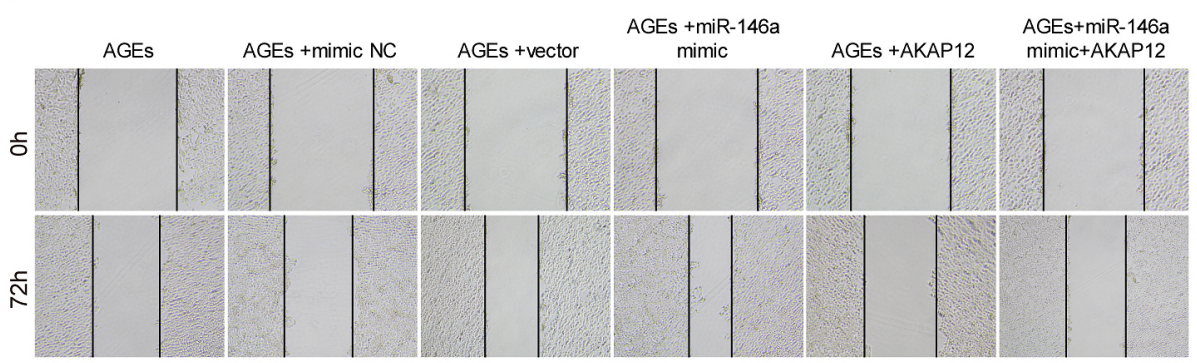

E

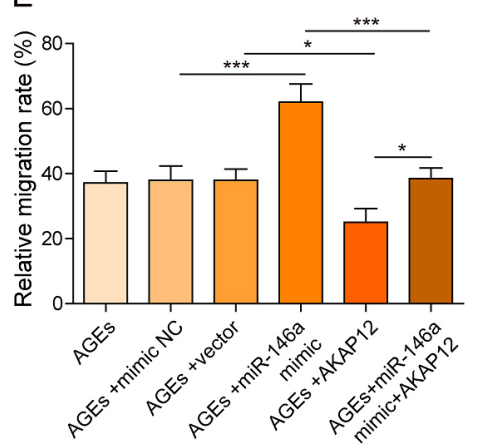

Fig. 5 Co-overexpression of miR-146a and AKAP12 reversed the suppression of cell proliferation and migration in an in vitro DFU model. (A and B) miR-146a and AKAP12 expression was measured by qRT-PCR. (C) The expression of AKAP12, HIF-1 $\alpha$, Wnt3a and $\beta$-catenin after overexpression of miR-146a and $A K A P 12$ was measured by western blotting in HaCaT cells. (D) The effect of co-overexpression of miR-146a and AKAP12 on the proliferation of HaCaT cells measured by MTT. (E) The effect of co-overexpression of miR-146a and AKAP12 on cell migration in an in vitro DFU model was measured by a cell scratch assay. $n=3 . * p<0.05, * * p<0.01, * * * p<0.001$.

was decreased and that AKAP12 expression was increased in an in vitro DFU model. Overexpression of miR-146a promoted cell proliferation and migration in an in vitro DFU model. There were no significant differences between AGE treatment and high glucose treatment of cells. Thus, this in vitro DFU model induced by AGEs was used for further analysis.

As an essential angiogenic regulator in endothelial cells, AKAP12 plays a vital role in the development of angiogenesis and wound healing [31, 35]. In our study, it was first confirmed that AKAP12 regulated cell prolife- ration and migration in $\mathrm{HaCaT}$ cells to promote wound healing. The results of the luciferase reporter assay revealed that miR-146a could directly target $A K A P 12$ in the in vitro DFU model. In addition, the overexpression of miR-146a suppressed AKAP12 and thus promoted cell proliferation and migration in vitro models. Our results suggested that miR-146a may be involved in cell proliferation and migration by targeting AKAP12 in an in vitro DFU model.

Furthermore, we explored the effect of cooverexpressed miR-146a and AKAP12 on cell proliferation 
and migration in an in vitro DFU model on the $\beta$-catenin signalling pathway. As described in a previous study, $\beta$ catenin plays an important role in wound healing via the Wnt signalling pathway, and the activation of $\beta$-catenin can promote the proliferation and migration of the $\mathrm{HaCaT}$ cell line in the skin lesion model induced by $\mathrm{H}_{2} \mathrm{O}_{2}$ [32, 34]. Similarly, overexpression of miR-146a regulated the activation of the $\beta$-catenin signalling pathway and promoted the proliferation and migration of HaCaT cells. In our study, suppression of AKAP12 significantly promoted the protein expressions of HIF-1 $\alpha$, $\mathrm{Wnt3a}$ and $\beta$-catenin. Moreover, the co-overexpression of miR-146a and AKAP12 in the in vitro DFU model reversed the activation of the $\beta$-catenin signalling pathway by miR-146a overexpression. When miR-146a was overexpressed, the expression levels of HIF-1 $\alpha$, Wnt3a and $\beta$-catenin were all increased. However, cooverexpression of miR-146a and AKAP12 reversed the expression levels of HIF- $1 \alpha$, Wnt3a and $\beta$-catenin, which were all significantly decreased. Our results suggested that AKAP12 may affect the Wnt/ $\beta$-catenin pathway by regulating HIF-1 $\alpha$, thereby affecting the development of DFU. Overexpression of miR-146a promoted cell proliferation and migration in a DFU model by suppressing AKAP12.

In conclusion, miR-146a could directly target AKAP12. Overexpression of miR-146a could promote cell proliferation and migration in an in vitro DFU model by regulating the AKAP12 axis. Our study provides a new perspective for the study of miR-146a treatment of DFU.

\section{Acknowledgments}

None.

\section{Disclosure}

The authors declare that they have no competing interests.

\section{References}

1. Lechner A, Akdeniz M, Tomova-Simitchieva T, Bobbert $\mathrm{T}$, Moga A, et al. (2019) Comparing skin characteristics and molecular markers of xerotic foot skin between diabetic and non-diabetic subjects: an exploratory study. $J$ Tissue Viability 28: 200-209.

2. Sørensen MLB, Jansen RB, Fabricius TW, Jørgensen B, Svendsen OL (2019) Healing of diabetic foot ulcers in patients treated at the Copenhagen Wound Healing Center in 1999/2000 and in 2011/2012. J Diabetes Res 2019: 6429575.

3. Yu P, Guo J, Li J, Chen W, Zhao T (2019) Co-expression network analysis revealing the key lncRNAs in diabetic foot ulcers. Arch Med Sci 15: 1123-1132.

4. Yuan L, Sun Y, Xu M, Zeng F, Xiong X (2019) miR-203 acts as an inhibitor for epithelial-mesenchymal transition process in diabetic foot ulcers via targeting interleukin-8. Neuroimmunomodulation 26: 239-249.

5. Adeghate J, Nurulain S, Tekes K, Feher E, Kalasz H, et al. (2017) Novel biological therapies for the treatment of diabetic foot ulcers. Expert Opin Biol Ther 17: 979-987.

6. Vanlerberghe B, Devemy F, Duhamel A, Guerreschi P, Torabi D (2014) [Conservative surgical treatment for diabetic foot ulcers under the metatarsal heads. A retrospective case-control study]. Ann Chir Plast Esthet 59: 161169 (In French).

7. Chen CY, Rao SS, Ren L, Hu XK, Tan YJ, et al. (2018) Exosomal DMBT1 from human urine-derived stem cells facilitates diabetic wound repair by promoting angiogenesis. Theranostics 8: 1607-1623.

8. Wetzler C, Kampfer H, Stallmeyer B, Pfeilschifter J, Frank S (2000) Large and sustained induction of chemo- kines during impaired wound healing in the genetically diabetic mouse: prolonged persistence of neutrophils and macrophages during the late phase of repair. J Invest Dermatol 115: 245-253.

9. Moura LI, Cruz MT, Carvalho E (2014) The effect of neurotensin in human keratinocytes-implication on impaired wound healing in diabetes. Exp Biol Med 239: 6-12.

10. Chen R, Chen QT, Dong YH (2019) Clinical efficacy of apatinib in treating metastatic gastric cancer and its effect on IL-17. Oncol Lett 17: 5447-5452.

11. Wu X, Huang S (2019) HER2-specific chimeric antigen receptor-engineered natural killer cells combined with apatinib for the treatment of gastric cancer. Bull Cancer 106: 946-958.

12. Xu Z, Hu C, Chen S, Zhang C, Yu J, et al. (2019) Apatinib enhances chemosensitivity of gastric cancer to paclitaxel and 5-fluorouracil. Cancer Manag Res 11: 4905-4915.

13. Wu R, Liao Y, Shen W, Liu Y, Zhang J, et al. (2018) Overexpression of Wilms' tumor 1 in skin lesions of psoriasis is associated with abnormal proliferation and apoptosis of keratinocytes. Mol Med Rep 18: 3973-3982.

14. Muniandy K, Gothai S, Tan WS, Kumar SS, Mohd Esa N, et al. (2018) In vitro wound healing potential of stem extract of Alternanthera sessilis. Evid Based Complement Alternat Med 2018: 3142073.

15. Raj D, Brash DE, Grossman D (2006) Keratinocyte apoptosis in epidermal development and disease. $J$ Invest Dermatol 126: 243-257.

16. $\mathrm{Xu} \mathrm{J}, \mathrm{Wu} \mathrm{W}$, Zhang L, Dorset-Martin W, Morris MW, et al. (2012) The role of microRNA-146a in the pathogenesis of the diabetic wound-healing impairment: correction with 
mesenchymal stem cell treatment. Diabetes 61: 2906 2912.

17. Chen J, Jiang Q, Jiang XQ, Li DQ, Jiang XC, et al. (2019) miR-146a promoted breast cancer proliferation and invasion by regulating NM23-H1. J Biochem 167: 41-48.

18. Ouyang Y, Fu X, Tan D, Peng S, Fu L (2019) [Expressions of miR-146a and miR-155 in different samples of chronic hepatitis B patients]. Zhong Nan Da Xue Xue Bao Yi Xue Ban 44: 845-849 (In Chinese).

19. Qiu M, Li T, Wang B, Gong H, Huang T (2020) miR-146a-5p regulated cell proliferation and apoptotic by targeting SMAD3 and SMAD4. Protein Pept Lett 27: 411-418.

20. Wang L, Zhang H, Lei D (2019) microRNA-146a promotes growth of acute leukemia cells by downregulating ciliary neurotrophic factor receptor and activating JAK2/ STAT3 signaling. Yonsei Med J 60: 924-934.

21. Guan YJ, Li J, Yang X, Du S, Ding J, et al. (2018) Evidence that miR-146a attenuates aging- and traumainduced osteoarthritis by inhibiting Notch1, IL-6, and IL-1 mediated catabolism. Aging Cell 17: e12752.

22. Liang $\mathrm{YC}, \mathrm{Wu} \mathrm{YP}, \mathrm{Li} \mathrm{XD}, \mathrm{Chen} \mathrm{SH}, \mathrm{Ye} \mathrm{XJ}$, et al. (2019) TNF-alpha-induced exosomal miR-146a mediates mesenchymal stem cell-dependent suppression of urethral stricture. J Cell Physiol 234: 23243-23255.

23. Feng J, Dong C, Long Y, Mai L, Ren M, et al. (2019) Elevated Kallikrein-binding protein in diabetes impairs wound healing through inducing macrophage M1 polarization. Cell Commun Signal 17: 60.

24. Huijberts MSP, Schaper NC, Schalkwijk CG (2010) Advanced glycation end products and diabetic foot disease. Diabetes Metab Res Rev 24: S19-S24.

25. Anderson MM, Heinecke JW (2003) Production of $\mathrm{N} \sim \varepsilon_{-}^{-}$ (Carboxymethyl) Lysine is impaired in mice deficient in NADPH oxidase: a role for phagocyte-derived oxidants in the formation of advanced glycation end products during inflammation. Diabetes 52: 2137-2143.

26. Monlun M, Hugo M, Blanco L, Mohammedi K, Ahdab R, et al. (2018) Nerve action potential amplitude, a robust marker of diabetic peripheral neuropathy. Diabet Med 35: 1460-1461.

27. Birgin E, Gebhardt C, Hetjens S, Fischer S, Rückert F, et al. (2018) Extracorporal shock wave therapy enhances receptor for advanced glycated end-product-dependent flap survival and angiogenesis. Ann Plast Surg 80: 424431.

28. Sharma Y, Saxena S, Mishra A, Saxena A, Natu SM (2013) Advanced glycation end products and diabetic retinopathy. J Ocul Biol Dis Infor 5: 63-69.
29. Zhou L, Wei W, Yang C, Zeng T, Hu M, et al. (2018) GADD45a promotes active DNA demethylation of the MMP-9 promoter via base excision repair pathway in AGEs-treated keratinocytes and in diabetic male rat skin. Endocrinology 159: 1172-1186.

30. Tuttle KR, Anderberg RJ, Cooney SK, Meek RL (2009) Oxidative stress mediates protein kinase $\mathrm{C}$ activation and advanced glycation end product formation in a mesangial cell model of diabetes and high protein diet. Am J Nephrol 29: $171-180$.

31. Steagall RJ, Rusiñol AE, Truong QA, Han Z (2006) HSPA12B is predominantly expressed in endothelial cells and required for angiogenesis. Arterioscler Thromb Vasc Biol 26: 2012-2018.

32. Yang HL, Tsai YC, Korivi M, Chang CT, Hseu YC (2017) Lucidone promotes the cutaneous wound healing process via activation of the PI $3 \mathrm{~K} / \mathrm{AKT}$, Wnt/ $\beta$-catenin and NF$\mathrm{B}$ signaling pathways. Biochim Biophys Acta 1864: 151168.

33. Zeng LF, Xiao Y, Sun L (2019) A glimpse of the mechanisms related to renal fibrosis in diabetic nephropathy. $A d v$ Exp Med Biol 1165: 49-79.

34. Ma M, Fu B, Yang X, Xiao Y, Pan M (2019) Adipose mesenchymal stem cell-derived exosomes promote cell proliferation, migration, and inhibit cell apoptosis via Wnt/ $/$-catenin signaling in cutaneous wound healing. $J$ Cell Biochem 120: 10847-10854.

35. Choi YK, Kim JH, Kim WJ, Lee HY, Park JA, et al. (2007) AKAP12 regulates human blood-retinal barrier formation by downregulation of hypoxia-inducible factor-1alpha. J Neurosci 27: 4472-4481.

36. Boso D, Rampazzo E, Zanon C, Bresolin S, Maule F, et al. (2019) HIF-1 $\alpha /$ Wnt signaling-dependent control of gene transcription regulates neuronal differentiation of glioblastoma stem cells. Theranostics 9: 4860-4877.

37. Lin CJ, Lan YM, Ou MQ, Ji LQ, Lin SD (2019) Expression of miR-217 and HIF-1 $\alpha /$ VEGF pathway in patients with diabetic foot ulcer and its effect on angiogenesis of diabetic foot ulcer rats. $J$ Endocrinol Invest 42: 13071317.

38. Habibi F, Soufi FG, Ghiasi R, Khamaneh AM, Alipour MR (2016) Alteration in inflammation-related miR-146a expression in NF-KB signaling pathway in diabetic rat hippocampus. Adv Pharm Bull 6: 99-103.

39. Luo J, Si ZZ, Li T, Li JQ, Zhang ZQ, et al. (2019) MicroRNA-146a-5p enhances radiosensitivity in hepatocellular carcinoma through replication protein A3-induced activation of the DNA repair pathway. Am J Physiol Cell Physiol 316: C299-C311. 